\section{Arthroscopic treatment of TMJ disorders}

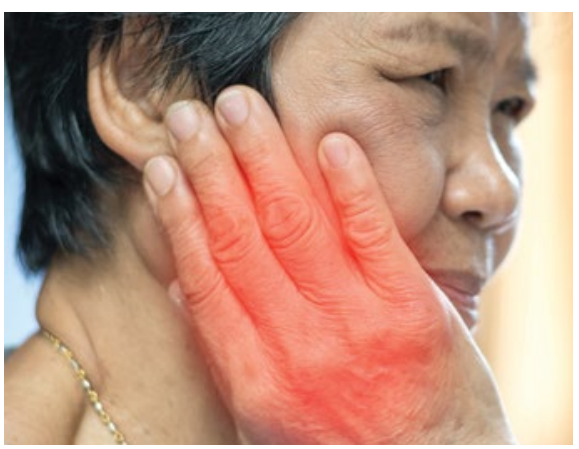

Where patients require mid-line treatment for TMJ disorders, finding the most minimally invasive solution is important.

Incito Medtech offers Arthroscope Systems for this reason. Using only the highest quality of materials with instruments that have been carefully designed for both surgical efficiency and patient comfort, the systems provide the tools professionals need to ensure safe and effective arthroscopic surgery.
TMJ disorders can have a massive impact on patients' lives. Where appropriate, offering as conservative a surgery as possible to correct their problems will encourage treatment acceptance and ensure they get the care they need.

For more information about the Arthroscope Systems, call Karen Joy on 07468 420496 or email Karen.Joy@incitomedtech. co.uk or visit http://incitomedtech.co.uk.

\title{
Perfect for all dentine retention pulpal pin requirements
}

FILPIN is the perfect product for all dentine retention pulpal pin requirements that provides maximum advantages without compromising safety, dentine integrity or retention. It is $99.8 \%$ pure titanium, more flexible, biocompatible and compatible with all dental materials. The self-threading, self-aligning pin speeds and eases placement for self-shearing first time, every time once optimum depth is reached. Its unique thread design maximises retention strength. After insertion FILPIN can be easily bent to suit the restoration without breaking it or the tooth. If minimal tooth structure is present pins are recommended to enhance chemical bonding provided by adhesives and when core build-up is used with less than one half of coronal tooth structure remaining. Bonding alone is not sufficient in many situations where minimal tooth structure is present. Pins provide anti-rotational benefit when a single post is used. Filpin shanks are autoclavable and may be reloaded many times with Filpin Spare Pin inserts. Available for use by handpiece or hand placement in two sizes together with a drill to complement each size. FILPOST is the only prefabricated post system that can be customised to suit the restoration for root post and core build up.
It can be bent and shortened without risk of fracture, enabling easy insertion of multiple posts into converging canals. There is more preserving of healthy tooth structure and it is stronger in use via its unique passive 'interlocking' system. Save time by using FILPOST as no drilling is required during placement thus avoiding risk of perforation. Its anatomical shape minimises dentine removal. Retention grooves along the post, working together with retention grooves formed within the canal surface, by the special Universal Groover, create a unique passive interlock that strengthens retention.

\section{Enhanced CPD for all}

The British Dental Conference and Dentistry Show offers hours of enhanced CPD designed for the entire dental team. Lecture programmes are dedicated to each role in the profession and sessions are led by nationally and internationally renowned speakers.

Further still, the trade floor will host more than 450 manufacturers and suppliers, with many providing on-stand learning opportunities as well as exclusive promotions and live demonstrations.

All this makes the event the perfect place to bring the whole team. Make sure you don't miss out for 2020 !
The British Dental Conference and Dentistry Show 2020 - 15 and 16 May Birmingham NEC, co-located with DTS.

For all the latest information, please visit www.thedentistryshow.co.uk, call 0207348 5270 or email dentistry@closerstillmedia. com.

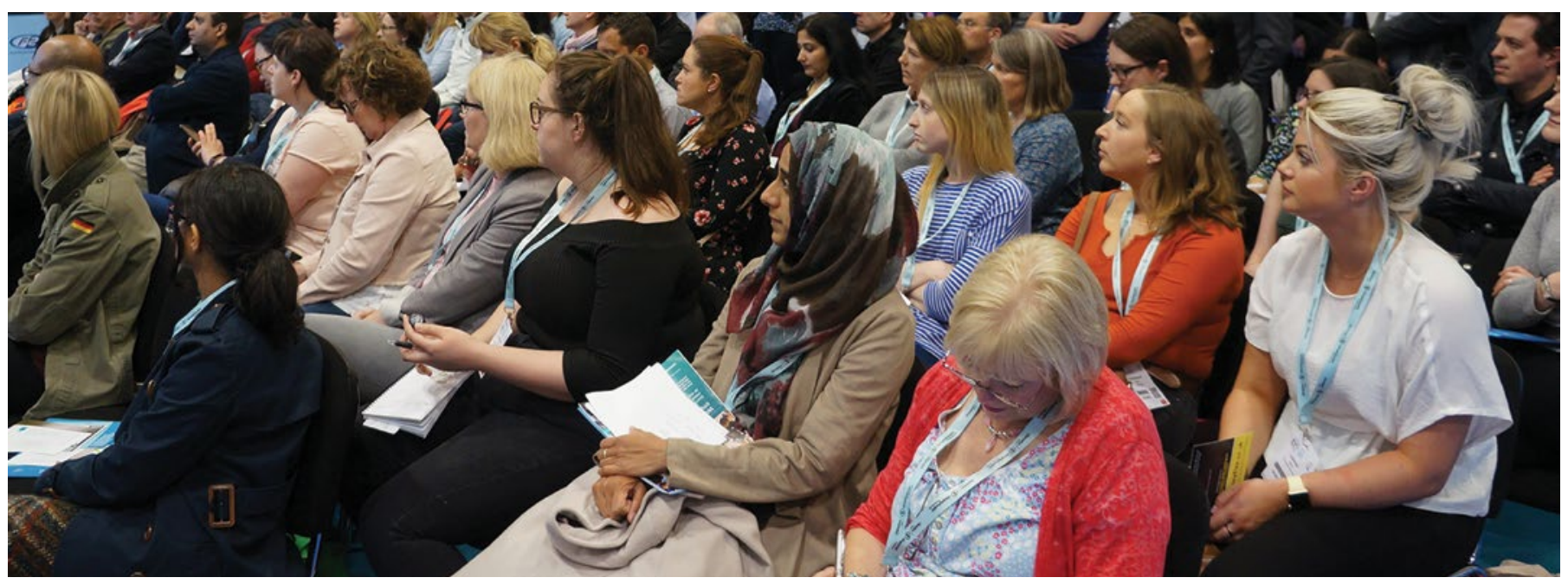

